\title{
In vitro analysis of drugs that improve hyperglycemia-induced blood- brain barrier dysfunction
}

\author{
Ryoma Kayano a , Yoichi Morofuji ${ }^{\text {b, }}{ }^{*}$, Shinsuke Nakagawa ${ }^{c}$, Shuji Fukuda ${ }^{\text {b }}$, \\ Daisuke Watanabe ${ }^{\mathrm{d}}$, Hiroki Ozawa ${ }^{\mathrm{a}}$, Masami Niwa ${ }^{\mathrm{d}, \mathrm{e}}$, Takayuki Matsuo ${ }^{\mathrm{b}}$ \\ a Department of Neuropsychiatry, Nagasaki University Graduate School of Biomedical Sciences, 1-7-1 Sakamoto, Nagasaki, 852-8501, Japan \\ b Department of Neurosurgery, Nagasaki University Graduate School of Biomedical Sciences, 1-7-1 Sakamoto, Nagasaki, 852-8501, Japan \\ c Department of Medical Pharmacology, Nagasaki University Graduate School of Biomedical Sciences, 1-12-4 Sakamoto, Nagasaki, 852-8523, Japan \\ d BBB Laboratory, PharmaCo-Cell Company, Ltd., Nagasaki, 1-43 Dejima, Nagasaki, 850-0862, Japan \\ e Nagasaki University, Japan
}

\section{A R T I C L E I N F O}

\section{Article history:}

Received 18 July 2018

Accepted 25 July 2018

Available online 29 July 2018

\section{Keywords:}

Blood-brain barrier

Candesartan

Hyperglycemia

Pitavastatin

\begin{abstract}
A B S T R A C T
Background: Blood-brain barrier (BBB) disruptions are a key feature of hyperglycemia (HG)-induced cerebral damage. Patients with diabetes mellitus often have other cerebrovascular disease risk factors including hypertension, dyslipidemia, arrhythmia, and atherosclerosis obliterans. However, whether the drugs for these comorbidities are effective for improving HG-induced BBB damage is unclear.

Methods: We investigated the effect of pitavastatin, candesartan, cilostazol, propranolol, and eicosapentaenoic acid on HG-induced BBB damage. In vitro BBB models consisting of primary cultures of rat brain capillary endothelial cells were subjected to HG (55 mM D-glucose).

Results: We observed a significant decrease in transendothelial electrical resistance (TEER) with HG, showing that HG compromised the integrity of the in vitro BBB model. No significant decrease in cell viability was seen with HG, but HG increased the production of reactive oxygen species. Pitavastatin and candesartan inhibited decreases in TEER induced by HG.

Conclusions: In summary, pitavastatin and candesartan improved HG-induced BBB damage and this in vitro model of $\mathrm{HG}$-induced $\mathrm{BBB}$ dysfunction contributes to the search for BBB protective drugs.
\end{abstract}

๑) 2018 Elsevier Inc. All rights reserved.

\section{Introduction}

The blood-brain barrier (BBB) acts as a dynamic interface between peripheral fluids and the central nervous system. Morphologically, the BBB consists of brain capillary endothelial cells joined by tight junctions. In addition, as brain capillary endothelial cells express specific transporters and receptors, the BBB is not only a barrier, but also a very specific and highly differentiated system for maintenance of brain homeostasis [1]. Therefore, pathological BBB dysfunction may disrupt the homeostasis of the neuronal environment and lead to neuronal damage [2].

Dysfunction of vascular endothelial cells is considered to be a key initial step in diabetic angiopathy that affects the progression and severity of diabetes. Diabetes induces microvascular complications in the brain by altering blood flow and BBB permeability

\footnotetext{
* Corresponding author.

E-mail address: yoichi51@hotmail.com (Y. Morofuji).
}

and inducing abnormal endothelial proliferation [3], thereby affecting cognitive function in diabetic individuals [4]. Several molecular mechanisms appear to be related to HG-induced endothelial dysfunction including increased polyol and hexosamine pathway activity, increased formation of advanced glycation endproducts, activation of protein kinase $\mathrm{C}$ isoforms, and increased reactive oxygen species (ROS) [5,6]. However, the mechanism involved in HG-induced BBB damage is still not fully understood.

In clinical setting, multiple drugs are often prescribed for diabetics as they are at high risk of cerebrovascular disease. Several reports have indicated that some drugs might improve brain damage caused by brain ischemia. The neuroprotective effects of cilostazol, statins, angiotensin II receptor blockers (ARB), and eicosapentaenoic acid (EPA) have been demonstrated in clinical studies [7-10]. However, whether they are effective for HGinduced BBB damage remains unclear. If these compounds can improve BBB function, new insight into understanding the mechanism of the protective effects of these compounds can be obtained. To test this hypothesis, we analyzed the effects of these compounds 
on HG-induced BBB damage by measuring transendothelial electrical resistance (TEER), a simple method for analyzing the barrier properties.

In the present study, we established an in vitro model of HGinduced BBB dysfunction and examined the effect of drugs which might have neuroprotective effects on barrier function of the BBB after exposure to HG.

\section{Materials and methods}

\subsection{Materials}

All reagents were purchased from Sigma (St. Louis, MO, USA) unless otherwise indicated. Wistar rats were obtained from Japan SLC, Inc. (Hamamatsu, Japan). All animals were treated in strict accordance with the National Institutes of Health Guide for the Care and Use of Laboratory Animals (NIH Publications No. 80-23).

\subsection{Primary cultures of rat brain capillary endothelial cells (RBECS) and construction of the in vitro BBB model}

Primary cultures of RBECs were prepared from 3-week-old rats, as described previously [11,12]. RBEC cultures were maintained in DMEM/F12 supplemented with 10\% fetal bovine plasma derived from serum (PDS) (Animal Technologies, Inc., Tyler, TX, USA), basic fibroblast growth factor $(1.5 \mathrm{ng} / \mathrm{mL}$; Roche Applied Sciences, Penzberg, Germany), heparin $(100 \mu \mathrm{g} / \mathrm{mL})$, insulin $(5 \mu \mathrm{g} / \mathrm{mL})$, transferrin $(5 \mu \mathrm{g} / \mathrm{mL})$, sodium selenite $(5 \mathrm{ng} / \mathrm{mL})$ (insulin-transferrin-sodium selenite medium supplement), gentamycin $(50 \mu \mathrm{g} /$ $\mathrm{mL})$, and puromycin $(4 \mu \mathrm{g} / \mathrm{mL})$ (RBEC I medium) at $37^{\circ} \mathrm{C}$ in a humidified atmosphere of $5.0 \% \mathrm{CO}_{2} / 95 \%$ air for 2 days. After 2 days, new medium containing all the components of RBEC medium except for puromycin (RBEC II medium) was added to the cells. To construct an in vitro model of the BBB, endothelial cells $\left(2.0 \times 10^{5}\right.$ cells $\left./ \mathrm{cm}^{2}\right)$ were seeded on the inside of inserts that were placed in the wells of 24-well culture plates. BBB models were maintained in RBEC medium II supplemented with $500 \mathrm{nM}$ hydrocortisone. In vitro BBB models were exposed to HG during RBEC growth.

\subsection{Treatment}

RBECs were exposed to $5.5 \mathrm{mM}$ D-glucose (WAKO Pure Chemical Ltd., Osaka, Japan) + 49.5 mM sucrose (WAKO Pure Chemical Ltd.), $27.5 \mathrm{mM}$ D-glucose $+27.5 \mathrm{mM}$ sucrose, $55 \mathrm{mM}$ D-glucose, or $55 \mathrm{mM}$ sucrose in RBEC II medium. Sucrose was used for normalization of osmotic effects. RBEC II only medium without addition of D-glucose or sucrose was added to cells as a control in Figs. 1A and 2A. After 24 and $48 \mathrm{~h}$, TEER was measured, as described below.

\subsection{Evaluation of tight junction function in RBECS}

To evaluate tight junction function in RBECs, TEER was measured using an EVOM resistance meter (World Precision Instruments, Sarasota, FL, USA). RBECs cultured on Millicell inserts in a 24-well plate were used to measure TEER. The resistance measurements of black filters (background resistance) were subtracted from filters with cells. The values are shown as $\Omega \times \mathrm{cm}^{2}$, and data indicate the rate of change in TEER before and after treatment compared to control.

\subsection{Paracellular permeability of sodium fluorescein}

After treatment for $48 \mathrm{~h}$, endothelial barrier function was evaluated by measuring the permeability of sodium fluorescein (Na-F,

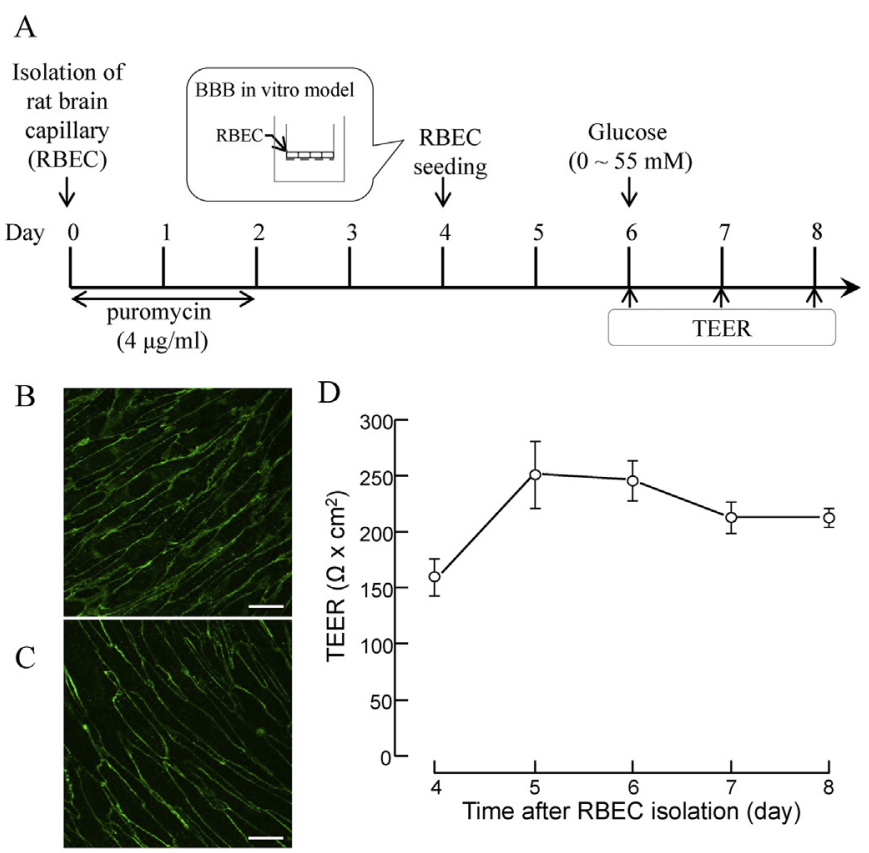

Fig. 1. Schematic drawing of the experiment with primary cultures of rat brain capillary endothelial cells (RBECs). (A) Flowchart of our in vitro blood-brain barrier (BBB) model. Primary cultures of RBECs were immunostained with the endothelial marker VE-cadherin (B) and the junctional protein marker occludin (C). Bar $=20 \mu \mathrm{m}$. (D) Typical TEER values of the BBB model. TEER in the BBB model reached more than $150 \Omega \times \mathrm{cm}^{2}$, and the plateau was maintained up to 8 days.

MW 376). To initiate the permeability studies, the medium in the luminal chamber of the insert $(0.2 \mathrm{~mL})$ was removed and replaced with assay buffer $\left(136 \mathrm{mM} \mathrm{NaCl}, 0.9 \mathrm{mM} \mathrm{CaCl}, 0.5 \mathrm{mM} \mathrm{MgCl}_{2}\right.$, $2.7 \mathrm{mM} \mathrm{KCl}, 1.5 \mathrm{mM} \mathrm{KH}_{2} \mathrm{PO}_{4}, 10 \mathrm{mM} \mathrm{NaH}_{2} \mathrm{PO}_{4}, 25 \mathrm{mM}$ glucose, and $10 \mathrm{mM}$ Hepes, pH 7.4) containing $10 \mu \mathrm{g} / \mathrm{mL}$ Na-F. Samples were removed from the abluminal chamber after $30 \mathrm{~min}$. The concentration of Na-F was determined using a fluorescence multi-well plate reader $(\operatorname{Ex}(\lambda) 485 \mathrm{~nm} ; \operatorname{Em}(\lambda) 535 \mathrm{~nm}$, Wallac 1420 ARVO Multilabel Counter, Perkin Elmer, Waltham, MA, USA). The permeability coefficient was calculated as previously described [13].

\subsection{Immunostaining}

To observe markers of brain endothelial cells, cells were stained with primary antibodies for VE-cadherin and occludin (1:200 each). Secondary antibodies were Alexa Fluor 488-conjugated donkey anti-mouse immunoglobulins (Invitrogen Corporation, Carlsbad, CA, USA, 1:1000). After culturing for 3 days on a culture slide, RBECs were washed in phosphate-buffered saline (PBS), fixed in 3\% paraformaldehyde in PBS for $10 \mathrm{~min}$, and permeabilized with $0.1 \%$ Triton-X 100 for 10 min. Cells were blocked with $3 \%$ bovine serum albumin and incubated with the primary antibodies anti-VEcadherin (Santa Cruz Biotechnology, Dallas, TX, USA) and antioccludin (Zymed Laboratories, South San Francisco, CA, USA) overnight at $4{ }^{\circ} \mathrm{C}$. Cells were rinsed three times with PBS and then incubated for $1 \mathrm{~h}$ at room temperature with secondary antibodies (Alexa Fluor 488-conjugated donkey anti-mouse immunoglobulins). Preparations were mounted in Gel Mount (Biomeda, Foster City, CA, USA), and staining was examined using a Zeiss LSM $5 \mathrm{~Pa}$ Confocal laser scanning microscope (Carl Zeiss AG, Okerkochen, Germany). 
A

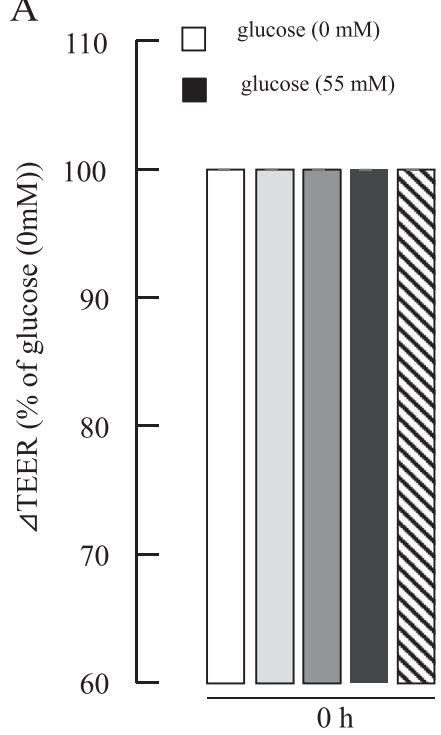

glucose $(5.5 \mathrm{mM})$

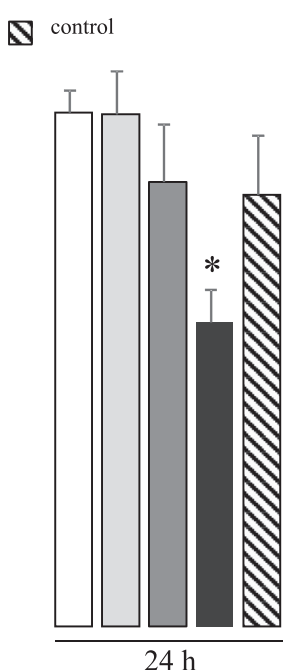

glucose $(27.5 \mathrm{mM})$

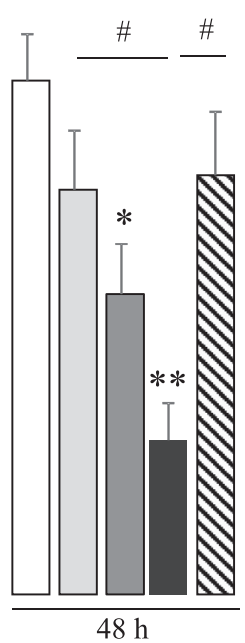

B

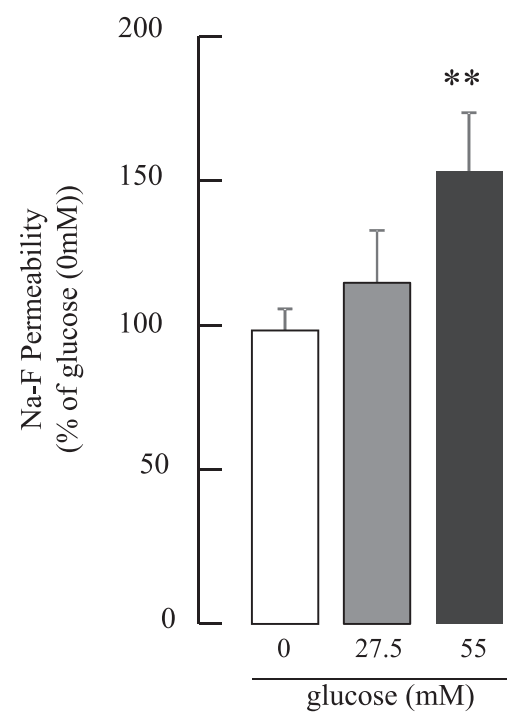

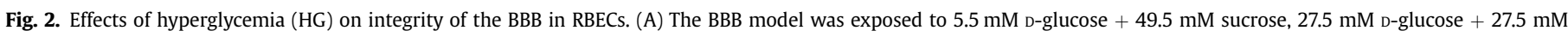

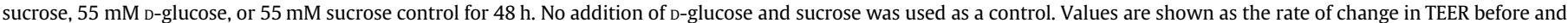

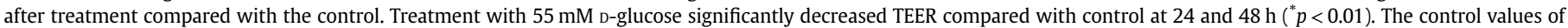

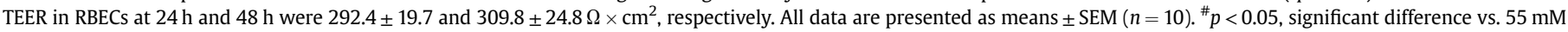

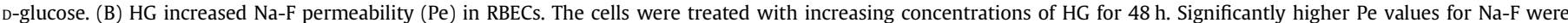

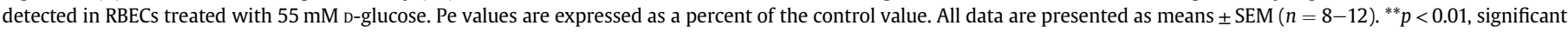
difference vs. $0 \mathrm{mM}$ D-glucose.

\subsection{Cell viability}

The Cell Counting Kit (CCK)-8 (Dojindo Laboratories, Kumamoto, Japan) assay was used to determine cell viability. RBECs were seeded in the wells of a 96-well plate and treated with sucrose and/ or D-glucose. After $48 \mathrm{~h}$, the medium was replaced with $200 \mu \mathrm{l}$ PBS containing $20 \mu \mathrm{l} \mathrm{CCK}-8$ for $60 \mathrm{~min}$ at $37^{\circ} \mathrm{C}$. Absorbance was measured at $450 \mathrm{~nm}$ (Wallac 1420 ARVO Multilabel Counter).

\subsection{Intracellular levels of ROS}

To measure the production of ROS, 5-(and-6)-chloromethyl$2^{\prime}, 7^{\prime}$-dichlorodihydrofluorescein diacetate, acetyl ester (CM$\mathrm{H}_{2}$ DCFDA, Invitrogen) was used. RBECs $\left(5 \times 10^{4} / \mathrm{cm}^{2}\right)$ were seeded into 96-well plates. Cells were pre-incubated with $0.1 \mathrm{~mL}$ assay buffer containing $10 \mu \mathrm{M} \mathrm{CM}-\mathrm{H}_{2}$ DCFDA for $1 \mathrm{~h}$, washed twice with assay buffer, and exposed to D-glucose $(0-55 \mathrm{mM})$ for $1 \mathrm{~h}$. Fluorescence intensity derived from $\mathrm{CM}-\mathrm{H}_{2}$ DCFDA was determined using a fluorescence multi-well plate reader $(\operatorname{Ex}(\lambda) 485 \mathrm{~nm} ; \operatorname{Em}(\lambda)$ $535 \mathrm{~nm}$ ). Cellular protein was measured with a BCA protein assay kit, and the data were normalized to protein content.

\subsection{Analysis of effects of pitavastatin, candesartan, cilostazol, and EPA after exposure to $H G$}

RBECs were exposed to $55 \mathrm{mM}$ D-glucose or $55 \mathrm{mM}$ sucrose in RBEC II medium. Pitavastatin (10 nM), candesartan $(10 \mathrm{nM})$, cilostazol $(3 \mu \mathrm{M})$, propranolol $(10 \mu \mathrm{M})$, or EPA $(50 \mu \mathrm{M})$ was added to the luminal (upper) compartment of the BBB model. TEER was measured after $48 \mathrm{~h}$. The concentration of each drug was determined according to previous publications.

\subsection{Statistical analysis}

All data are expressed as means \pm standard error of the mean
(SEM). Values were compared using analysis of variance followed by the Tukey-Kramer method. A $p$ value of less than 0.05 was considered to be statistically significant.

\section{Results}

\subsection{Construction of the in vitro BBB model}

Brain endothelial cells are the major components of the BBB. Fig. 1 shows the characteristics of the cultures employed in the present study. Confluent endothelial cells expressed VE-cadherin, a marker of endothelial cells (Fig. 1B). The tight junction protein occludin, which is specifically expressed by the brain type of endothelial cells, was strongly expressed and concentrated primarily at cell-cell borders (Fig. 1C). Furthermore, TEER of the RBEC monolayers used in the present study was more than $150 \Omega \times \mathrm{cm}^{2}$, indicating formation of a functional barrier (Fig. 1D).

\subsection{Effects of HG on the cerebral barrier}

To analyze the potentially harmful effects of HG on BBB integrity, we measured TEER in cells exposed to $5.5 \mathrm{mM}$ D-glucose + $49.5 \mathrm{mM}$ sucrose, $27.5 \mathrm{mM}$ D-glucose $+27.5 \mathrm{mM}$ sucrose, $55 \mathrm{mM}$ Dglucose, or $55 \mathrm{mM}$ sucrose control. Treatment with $55 \mathrm{mM}$ Dglucose significantly decreased the TEER compared with control at 24 and $48 \mathrm{~h}$ (TEER (\% of control); control: $100 \pm 1.7$ vs. $55 \mathrm{mM}$ Dglucose: $83.7 \pm 2.5 ; p<0.01,24 \mathrm{~h}$; control: $100 \pm 3.6$ vs. $55 \mathrm{mM}$ Dglucose: $72.0 \pm 2.9 ; p<0.01,48 \mathrm{~h}$ ) (Fig. $2 \mathrm{~A}$ ). Because the strongest effects of HG were observed with $55 \mathrm{mM}$ D-glucose, this concentration was used in subsequent experiments. HG also affected the paracellular permeability of Na-F, a small water-soluble marker, across the RBEC monolayer at $48 \mathrm{~h}$ after treatment with HG (Fig. 2B). HG treatment $(55 \mathrm{mM})$ significantly increased the permeability of Na-F in RBECs to $154.1 \pm 19.8 \%$ of the control. Thus, HG compromised the barrier properties of the BBB in RBECs. 


\subsection{The change in cell number and ROS production after exposure to $H G$}

To investigate the effects of HG on cell viability and ROS production, we measured the cell number and ROS production using chemical compounds. No significant differences in cell number were observed between control and HG (Fig. 3A). HG affected the production of ROS in the RBEC monolayer. Treatment with $55 \mathrm{mM}$ D-glucose significantly increased the production of ROS in RBECs to $149.2 \pm 9.5 \%$ of the control (Fig. 3B). Thus, HG may compromise the barrier properties of the BBB in RBECs via ROS.

\subsection{Effect of cilostazol, pitavastatin, candesartan, propranolol, and EPA on barrier function in the BBB model}

We investigated the effects of cilostazol, pitavastatin, and candesartan, and EPA which have neuro-protective effects in vivo, against HG-induced BBB damage (Fig. 4). Spindler and Waschke reported that beta-adrenergic stimulation contributes to maintenance of endothelial barrier function [14]. To examine a compound that aggravates the HG-induced BBB dysfunction, we also examined the effects of propranolol, a beta-adrenergic receptor antagonist, on HG-induced BBB damage. Treatment with $10 \mathrm{nM}$ pitavastatin and $10 \mathrm{nM}$ candesartan significantly increased TEER after exposure to HG (TEER (\% of control); HG + pitavastatin: $92.1 \pm 4.4$ vs. HG: $70.3 \pm 1.4 ; p<0.01 ; \mathrm{HG}+$ candesartan: $86.9 \pm 3.5$ vs. HG $73.6 \pm 2.2$; $p<0.01$ ). Treatment with cilostazol, propranolol, and EPA did not significantly increase TEER under HG.

\section{Discussion}

In the present study, we found that HG compromised the BBB integrity in an in vitro model. Pitavastatin and candesartan improved HG-induced BBB damage.

Diabetes mellitus is associated with microvascular complications and increased risk of neurological events. Many clinical and animal studies have also suggested that BBB leakage is one of the major factors causing cognitive decline in diabetics. Srivastava et al. reported that HG compromises the structural and functional capacities of an in vitro model of the human cerebral barrier [15]. Starr et al. reported hyperpermeability of the BBB in patients with type II diabetes on gadolinium-enhanced magnetic resonance imaging [16]. In this study, we found that HG decreased TEER and increased the permeability of Na-F in our in vitro model. These findings support the hypothesis that HG compromises barrier integrity.

Shao and Bayraktutan reported that HG promotes human brain microvascular endothelial cell apoptosis via induction of protein kinase C- $\beta 1$ and the pro-oxidant enzyme NADPH oxidase [17]. The apoptotic effect of HG on endothelial cells derived from large arteries and the venous system (e.g., aorta and umbilical vein) is well documented $[18,19]$. However, in this study, no significant differences in the cell number were observed between control and HG. This discrepancy may have been due to differences in the species, incubation time, or cell culture conditions. HG activates the glycolytic pathway and increases the production of diacylglycerol [20]. Elevation of intracellular diacylglycerol levels activates protein kinase $C$ and subsequently activates NADPH oxidase to induce ROS [21]. We found that HG significantly increased the production of ROS in RBECs. Our results support the hypothesis that HG enhances ROS production. In our in vitro BBB model, these findings indicate that one mechanism of HG-induced BBB damage may be related to an increase in ROS production rather than a decrease in cell viability. Several groups have reported the effects of HG on properties of the BBB in vitro. Some groups have used immortalized cell lines to construct a BBB model [22,23], and others have used primary cultured cells $[15,24]$. Although primary cultures are expensive, time-consuming, and require expertise, primary cultured cells have the advantage of retaining the in vivo physiological characteristics, especially tight junction functions $[25,26]$. Thus, we used primary cultured cells for this study.

Improving BBB function during brain injury prevents the penetration of neurotoxic factors into the brain parenchyma and leads to neuronal survival. We analyzed clinically used drugs for patients with diabetes that may protect the BBB.
A

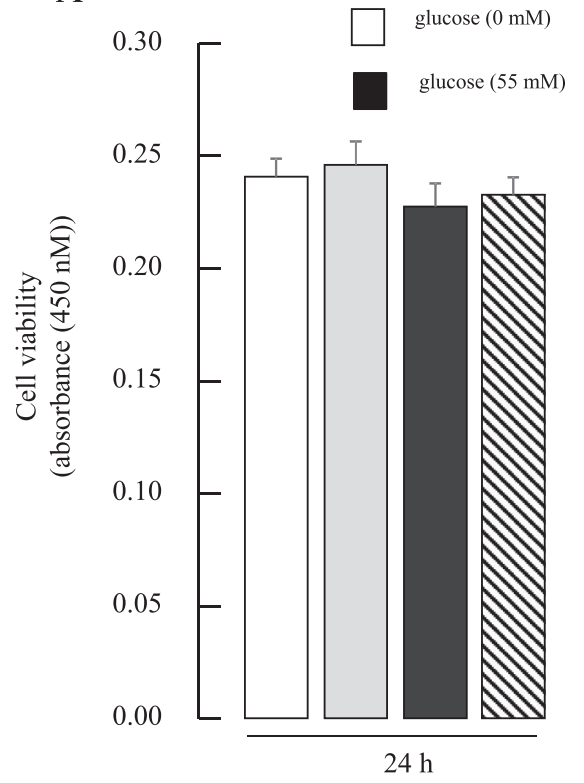

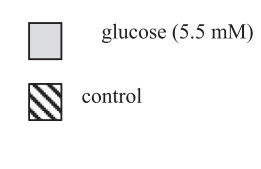

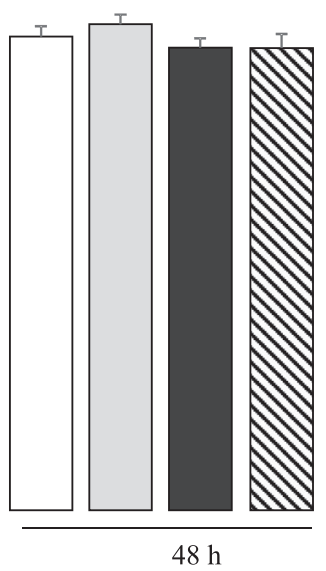

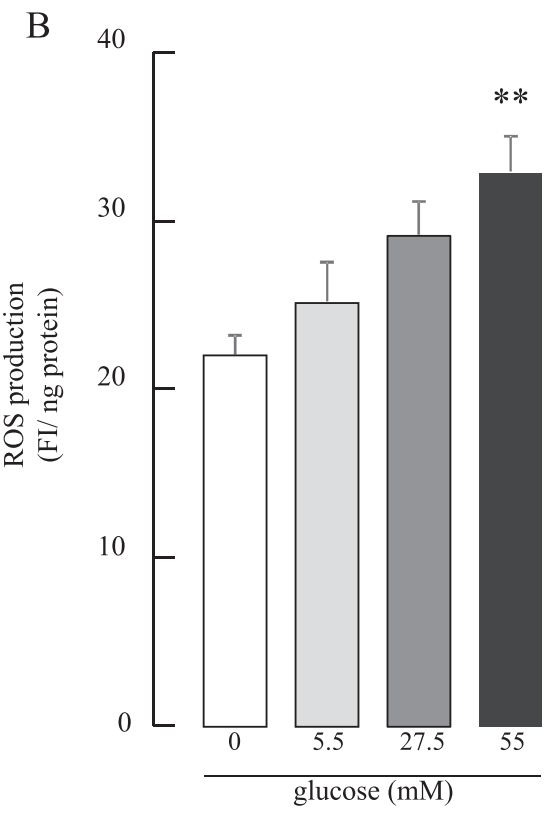

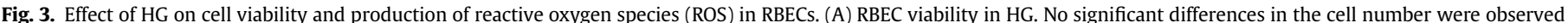

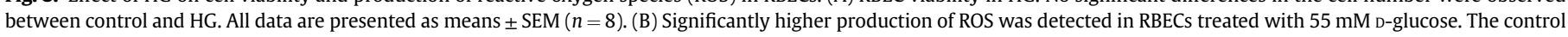
value for ROS production was $22.1 \pm 1.1$ arbitrary units (fluorescein intensity/ng protein). All data are presented as means \pm SEM $(n=5)$. ${ }^{* *} p<0.01$ vs. control. 
A
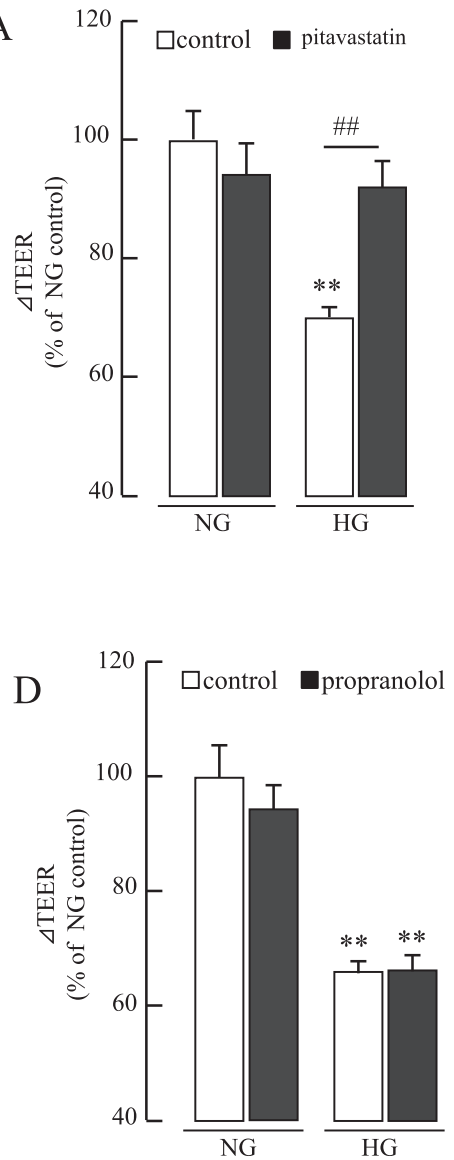

B

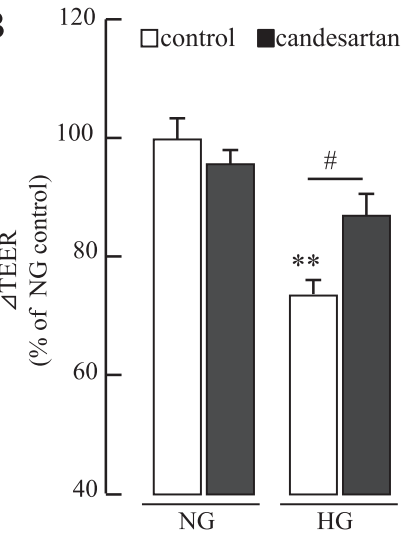

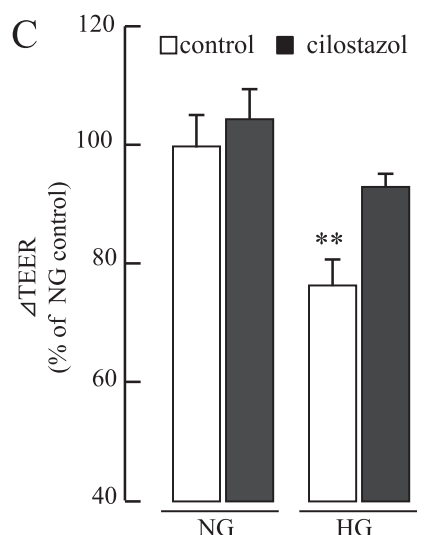

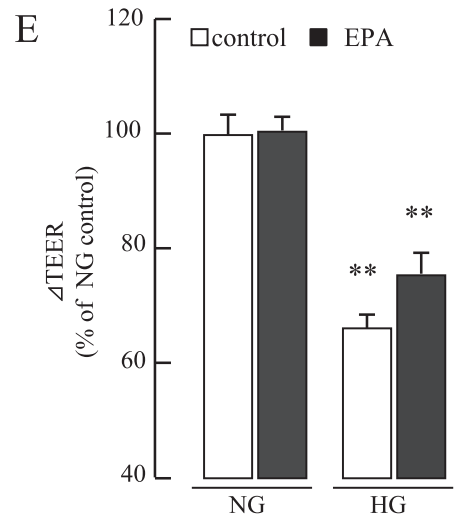

Fig. 4. Effects of (A) pitavastatin, (B) candesartan, (C) cilostazol, (D) propranolol, and (E) eicosapentaenoic acid (EPA) on TEER in the in vitro BBB model under normoglycemia (NG) and hyperglycemia (HG). Treatment with $10 \mathrm{nM}$ pitavastatin and $10 \mathrm{nM}$ candesartan significantly increased TEER after exposure to HG $\left({ }^{\#} p<0.05\right.$, $\left.{ }^{\# \#} p<0.01\right)$. Treatment with $3 \mu \mathrm{M}$ cilostazol, $10 \mu \mathrm{M}$ propranolol, and $50 \mu \mathrm{M}$ EPA did not significantly increase TEER. TEER values are expressed as a percentage of the control value. The control values for TEER in RBECs were $117.4 \pm 18.7$ (A, B), $233.5 \pm 23.8$ (C), and $158.4 \pm 9.8$ (D, E) $\Omega \times \mathrm{cm}^{2}$. All data are presented as means \pm SEM $(n=8-18)$. ${ }^{* *} p<0.01$ vs. normoglycemia control.

The neuroprotective and beneficial effect of statins has been demonstrated in animal models. Morofuji et al. reported that pitavastatin strengthens the barrier integrity in primary cultures of RBECs by up-regulating claudin 5 expression [27]. Riad et al. reported that low-dose atorvastatin leads to anti-oxidative and antiinflammatory effects in an animal model of diabetes [28]. In this study, we found that pitavastatin improved HG-induced BBB damage in our in vitro study. This finding might supports that the therapeutic efficacy of statins is due to the maintenance of BBB function to prevent cognitive function, vascular dementia, and Alzheimer's disease.

Many clinical and in vitro studies conclusively demonstrate the strong neuroprotective effects achieved by ARB administration [29]. The many clinical studies demonstrating beneficial effects of ARB administration in stroke, hypertension and diabetes have been described. So et al. demonstrated that candesartan improved ischemia-induced impairment of blood-brain barrier in vitro. In this study, we found that candesartan improved HG-induced BBB damage in our in vitro model [30]. This finding supports the hypothesis of cerebrovascular neuroprotective effects of ARBs in patients with diabetes.

The protective effect of cilostazol has been reported in animal models. cAMP plays an important role in stabilizing the barrier properties of endothelial cells [26]. Cilostazol is a selective inhibitor of phosphodiesterase type 3 , and accordingly, cilostazol increases intracellular cAMP levels and activates protein kinase A. Horai et al. reported that cilostazol elevates intracellular cAMP levels in RBECs and improves BBB integrity via the cAMP/protein kinase A pathway [31]. Takeshita et al. reported that cilostazol improves ischemiareperfusion injury enhanced by advanced glycation end-products by inhibiting tight junction protein disruption by reducing the effects of transforming growth factor- $\beta 1$ on brain capillary endothelial cells [32]. In this study, although cilostazol tended to attenuate HG-induced BBB damage, cilostazol did not significantly improve HG-induced BBB damage in our in vitro model. Cilostazol may not be associated with the mechanisms by which HG damages brain microvasculature. Spindler and Waschke reported that betaadrenergic stimulation contributes to maintenance of peripheral endothelial barrier function under baseline conditions [14]. As adrenergic $\beta_{2}$ receptor stimulation increases the intracellular cAMP level, $\beta_{2}$ agonists may improve barrier function. In addition, $\beta$ blockers can prolong, enhance, or alter the symptoms of hypoglycemia in diabetic patients. To examine a compound that aggravates HG-induced BBB dysfunction, we also examined the effects of propranolol, a beta-adrenergic receptor antagonist, on HG-induced BBB damage. However, propranolol did not affect the barrier function of the BBB in either normoglycemic or HG conditions.

Taneda et al. reported that EPA repairs diabetic tubular injury by regulating oxidative stress and mitochondrial apoptosis [33]. In this study, we found that EPA did not improve HG-induced BBB damage in our in vitro model. EPA may not be associated with the mechanisms by which HG damages the brain microvasculature.

In summary, $\mathrm{HG}$ compromised the integrity of the in vitro $\mathrm{BBB}$ model. Our HG-induced BBB dysfunction model will contribute to 
the search for BBB protective drugs. Pitavastatin and candesartan appeared to improve HG-induced BBB damage. The present study suggests that pitavastatin and candesartan may act as BBBprotective drugs in diabetic cerebral vessel disease.

\section{Funding}

This work was supported, in part, by Grant-in-Aid for Scientific Research (C) 26462167 (to S.N.) and (C) 17K10840 (to Y.M.) from the Japan Society for the Promotion of Science, Japan.

\section{Conflicts of interest}

All authors have no competing interests.

\section{Acknowledgment}

We would like to thank Kentaro Hayashi, Izumi Nagata for their critical reviews of the manuscript and helpful professional guidance.

\section{Transparency document}

Transparency document related to this article can be found online at https://doi.org/10.1016/j.bbrc.2018.07.131

\section{References}

[1] N.J. Abbott, A.A. Patabendige, D.E. Dolman, S.R. Yusof, D.J. Begley, Structure and function of the blood-brain barrier, Neurobiol. Dis. 37 (2010) 13-25.

[2] B.V. Zlokovic, The blood-brain barrier in health and chronic neurodegenerative disorders, Neuron 57 (2008) 178-201.

[3] B.T. Hawkins, T.F. Lundeen, K.M. Norwood, H.L. Brooks, R.D. Egleton, Increased blood-brain barrier permeability and altered tight junctions in experimental diabetes in the rat: contribution of hyperglycaemia and matrix metalloproteinases, Diabetologia 50 (2007) 202-211.

[4] M. Mogi, M. Horiuchi, Neurovascular coupling in cognitive impairment associated with diabetes mellitus, Circ. J. 75 (2011) 1042-1048.

[5] M. Brownlee, Biochemistry and molecular cell biology of diabetic complications, Nature 414 (2001) 813-820.

[6] S. Prasad, R.K. Sajja, P. Naik, L. Cucullo, Diabetes mellitus and blood-brain barrier dysfunction: an overview, J Pharmacovigil 2 (2014) 125.

[7] Y. Shinohara, Y. Katayama, S. Uchiyama, T. Yamaguchi, S. Handa, K. Matsuoka, Y. Ohashi, N. Tanahashi, H. Yamamoto, C. Genka, Y. Kitagawa, H. Kusuoka, K. Nishimaru, M. Tsushima, Y. Koretsune, T. Sawada, C. Hamada, group, Cilostazol for prevention of secondary stroke (CSPS 2): an aspirin-controlled, double-blind, randomised non-inferiority trial, Lancet Neurol. 9 (2010) 959-968.

[8] T. Kuwashiro, M. Kamouchi, T. Ago, J. Hata, H. Sugimori, T. Kitazono, The factors associated with a functional outcome after ischemic stroke in diabetic patients: the Fukuoka Stroke Registry, J. Neurol. Sci. 313 (2012) 110-114.

[9] M. Ueda, T. Inaba, C. Nito, N. Kamiya, Y. Katayama, Therapeutic impact of eicosapentaenoic acid on ischemic brain damage following transient focal cerebral ischemia in rats, Brain Res. 1519 (2013) 95-104.

[10] A. Nassief, J.D. Marsh, Statin therapy for stroke prevention, Stroke 39 (2008) 1042-1048.

[11] S. Nakagawa, M.A. Deli, S. Nakao, M. Honda, K. Hayashi, R. Nakaoke, Y. Kataoka, M. Niwa, Pericytes from brain microvessels strengthen the barrier integrity in primary cultures of rat brain endothelial cells, Cell. Mol. Neurobiol. 27 (2007) 687-694.

[12] S. Nakagawa, M.A. Deli, H. Kawaguchi, T. Shimizudani, T. Shimono, A. Kittel, K. Tanaka, M. Niwa, A new blood-brain barrier model using primary rat brain endothelial cells, pericytes and astrocytes, Neurochem. Int. 54 (2009) 253-263.

[13] K.A. Youdim, A. Avdeef, N.J. Abbott, In vitro trans-monolayer permeability calculations: often forgotten assumptions, Drug Discov. Today 8 (2003) 997-1003.

[14] V. Spindler, J. Waschke, Beta-adrenergic stimulation contributes to maintenance of endothelial barrier functions under baseline conditions, Microcirculation (N. Y.) 18 (2011) 118-127.

[15] K. Srivastava, B. Shao, U. Bayraktutan, PKC-beta exacerbates in vitro brain barrier damage in hyperglycemic settings via regulation of RhoA/Rho-kinase MLC2 pathway, J. Cerebr. Blood Flow Metabol. 33 (2013) 1928-1936.

[16] J.M. Starr, J. Wardlaw, K. Ferguson, A. MacLullich, I.J. Deary, I. Marshall, Increased blood-brain barrier permeability in type II diabetes demonstrated by gadolinium magnetic resonance imaging, J. Neurol. Neurosurg. Psychiatry 74 (2003) 70-76.

[17] B. Shao, U. Bayraktutan, Hyperglycaemia promotes human brain microvascular endothelial cell apoptosis via induction of protein kinase C-ssI and prooxidant enzyme NADPH oxidase, Redox Biol 2 (2014) 694-701.

[18] X.L. Du, G.Z. Sui, K. Stockklauser-Farber, J. Weiss, S. Zink, B. Schwippert, Q.X. Wu, D. Tschope, P. Rosen, Introduction of apoptosis by high proinsulin and glucose in cultured human umbilical vein endothelial cells is mediated by reactive oxygen species, Diabetologia 41 (1998) 249-256.

[19] H. Nakagami, R. Morishita, K. Yamamoto, S.I. Yoshimura, Y. Taniyama, M. Aoki, H. Matsubara, S. Kim, Y. Kaneda, T. Ogihara, Phosphorylation of p38 mitogenactivated protein kinase downstream of bax-caspase-3 pathway leads to cell death induced by high D-glucose in human endothelial cells, Diabetes 50 (2001) 1472-1481.

[20] B.A. Wolf, J.R. Williamson, R.A. Easom, K. Chang, W.R. Sherman, J. Turk, Diacylglycerol accumulation and microvascular abnormalities induced by elevated glucose levels, J. Clin. Invest. 87 (1991) 31-38.

[21] N. Koike, T. Takamura, S. Kaneko, Induction of reactive oxygen species from isolated rat glomeruli by protein kinase C activation and TNF-alpha stimulation, and effects of a phosphodiesterase inhibitor, Life Sci. 80 (2007) $1721-1728$.

[22] J. Yan, Z. Zhang, H. Shi, HIF-1 is involved in high glucose-induced paracellular permeability of brain endothelial cells, Cell. Mol. Life Sci. 69 (2012) 115-128.

[23] R.K. Sajja, S. Prasad, L. Cucullo, Impact of altered glycaemia on blood-brain barrier endothelium: an in vitro study using the hCMEC/D3 cell line, Fluids Barriers CNS 11 (2014) 8.

[24] C.L. Allen, U. Bayraktutan, Antioxidants attenuate hyperglycaemia-mediated brain endothelial cell dysfunction and blood-brain barrier hyperpermeability, Diabetes Obes. Metabol. 11 (2009) 480-490.

[25] S. Veszelka, A. Toth, F.R. Walter, A.E. Toth, I. Grof, M. Meszaros, A. Bocsik E. Hellinger, M. Vastag, G. Rakhely, M.A. Deli, Comparison of a rat primary cellbased blood-brain barrier model with epithelial and brain endothelial cel lines: gene expression and drug transport, Front. Mol. Neurosci. 11 (2018) 166.

[26] M.A. Deli, C.S. Abraham, Y. Kataoka, M. Niwa, Permeability studies on in vitro blood-brain barrier models: physiology, pathology, and pharmacology, Cell. Mol. Neurobiol. 25 (2005) 59-127.

[27] Y. Morofuji, S. Nakagawa, G. So, T. Hiu, S. Horai, K. Hayashi, K. Tanaka, K. Suyama, M.A. Deli, I. Nagata, M. Niwa, Pitavastatin strengthens the barrier integrity in primary cultures of rat brain endothelial cells, Cell. Mol. Neurobiol. 30 (2010) 727-735

[28] A. Riad, J. Du, S. Stiehl, D. Westermann, Z. Mohr, M. Sobirey, W. Doehner V. Adams, M. Pauschinger, H.P. Schultheiss, C. Tschope, Low-dose treatment with atorvastatin leads to anti-oxidative and anti-inflammatory effects in diabetes mellitus, Eur. J. Pharmacol. 569 (2007) 204-211.

[29] J.M. Saavedra, Beneficial effects of Angiotensin II receptor blockers in brain disorders, Pharmacol. Res. 125 (2017) 91-103.

[30] G. So, S. Nakagawa, Y. Morofuji, T. Hiu, K. Hayashi, K. Tanaka, K. Suyama, M.A. Deli, I. Nagata, T. Matsuo, M. Niwa, Candesartan improves ischemiainduced impairment of the blood-brain barrier in vitro, Cell. Mol. Neurobiol. 35 (2015) 563-572.

[31] S. Horai, S. Nakagawa, K. Tanaka, Y Morofuji, P.O Couraud, M.A. Deli, M. Ozawa, M. Niwa, Cilostazol strengthens barrier integrity in brain endothelial cells, Cell. Mol. Neurobiol. 33 (2013) 291-307.

[32] T. Takeshita, S. Nakagawa, R. Tatsumi, G. So, K. Hayashi, K. Tanaka, M.A. Deli, I. Nagata, M. Niwa, Cilostazol attenuates ischemia-reperfusion-induced bloodbrain barrier dysfunction enhanced by advanced glycation endproducts via transforming growth factor-beta1 signaling, Mol. Cell. Neurosci. 60 (2014) $1-9$.

[33] S. Taneda, K. Honda, K. Tomidokoro, K. Uto, K. Nitta, H. Oda, Eicosapentaenoic acid restores diabetic tubular injury through regulating oxidative stress and mitochondrial apoptosis, Am. J. Physiol. Ren. Physiol. 299 (2010) F1451-F1461. 